\title{
ORGANIZACIONES Y PROFESIONES EN LA SOCIEDAD CONTEMPORANEA
}

\section{Josep A. Rodríguez y Mauro F. Guillén}

RESUMEN. La sociedad post-industrial se caracteriza por ser una sociedad de profesiones y organizaciones. Las profesiones dominan la definición de los problemas y necesidades sociales y las actuaciones para hacerles frente, y las organizaciones son los espacios que articulan dichos procesos de definición y actuación. En este artículo se presentan las aproximaciones sociológicas más novedosas al estudio de organizaciones y profesiones como elementos centrales para el análisis y comprensión de la sociedad de finales del siglo $\mathrm{xx}$.

El desarrollo de las profesiones es una de las características fundamentales del siglo xx. Los avances en el conocimiento y la creciente complejidad de la sociedad occidental favorecen el desarrollo de las profesiones clásicas y la profesionalización de la sociedad. Un número cada vez mayor de parcelas de la vida social e individual se están convirtiendo en campos de conocimiento y de ejercicio de un creciente número de expertos profesionales. Goode (1960) ya planteaba hace varias décadas que una sociedad en industrialización se caracterizaba por ser una sociedad en profesionalización. La sociedad postindustrial actual, en la que destaca el enorme peso del sector servicios frente al sector industrial, se puede definir ya como una sociedad de profesiones. Somos testigos de un vertiginoso aumento de la proporción de las ocupaciones del sector servicios, y especialmente de profesiones y semiprofesiones. Junto a ello observamos cómo 
un número creciente de ocupaciones intentan adquirir los símbolos de estatus de las profesiones. El desarrollo de las profesiones pone sobre el tapete los cambios en la estructura de clases (y el desarrollo de una nueva clase media) y los cambios en el mercado de trabajo (con una drástica reducción de la clase trabajadora clásica y un gran aumento de las ocupaciones de servicios). La importancia de las profesiones y expertos en la estructura y dinámica de nuestra sociedad indican la capacidad de los sistemas de conocimiento profesional para la definición de viejos y nuevos problemas. El conocimiento abstracto se configura como un eficaz mecanismo para el mantenimiento de posiciones sociales de poder y privilegio.

Las organizaciones son los elementos fundamentales con los que se construye la sociedad actual, son los vehículos principales a través de los cuales se desarrolla la acción colectiva (Coleman, 1974). Por encima de todo, las organizaciones coordinan las acciones de las personas en la búsqueda de objetivos demasiado amplios para poder ser alcanzados individualmente. Cuando los grupos de intereses y las clases sociales realizan acciones colectivas, lo hacen utilizando organizaciones. Cualquier tipo de movimiento social tiene una mayor probabilidad de éxito si puede utilizar para su acción organizaciones ya existentes (Tilly, 1978). El Estado es en sí mismo un aparato organizacional. Las luchas por poder y control en las sociedades modernas implican el enfrentamiento entre organizaciones por conseguir posiciones privilegiadas en la estructura estatal, así como enfrentamientos entre el Estado y otros tipos de organizaciones. La concentración de poder en las organizaciones permite alcanzar objetivos a gran escala en las sociedades complejas y también permite algunas de las acciones más problemáticas que afectan a la sociedad. Las organizaciones se infiltran en nuestras vidas, y tendemos a considerar su existencia como algo dado. La mayor parte de las vidas de los individuos sólo se puede describir en términos de sus relaciones con organizaciones: nacido en un hospital, educado en escuelas, empleado por una empresa, cuidado por la organización sanitaria, e incluso entretenido por ciertas organizaciones. Progresivamente, además, un número mayor de tareas de nuestra sociedad son resueltas no por organizaciones individuales, sino por grupos de organizaciones en interdependencia. Dado que las organizaciones interpretan papeles cruciales en la sociedad moderna, la velocidad y dirección del cambio social depende de las dinámicas de las organizaciones. En concreto, la habilidad de la sociedad en su conjunto para responder a condiciones cambiantes depende del nivel de respuesta de las organizaciones que la forman y de la diversidad de sus poblaciones de organizaciones (Hannan y Freeman, 1989). 


\section{EL ESTUDIO DE LAS PROFESIONES EN LA SOCIEDAD CONTEMPORANEA}

El impacto de las profesiones en las organizaciones, sobre el Estado y sobre el conjunto de la sociedad ha atraído la atención de los grandes teóricos de la sociología desde sus orígenes. Las profesiones del siglo XIX estaban en su mayoría situadas fuera del corazón industrial de la sociedad. $\mathrm{Su}$ organización colegial jerarquizada no procede de la nueva forma organizativa capitalista, sino del Antiguo Régimen. Por ello, las profesiones fueron un gran enigma para los teóricos sociales. Marx intenta establecer el carácter de las clases profesionales, especialmente en su contribución negativa a la plusvalía. Contempla la especialización de los expertos como elemento básico para el desarrollo completo de la división del trabajo y del sistema de dominación de clases. Weber dedicó muchas páginas de su obra a intentar elucidar entre su racionalidad e irracionalidad. Como expertos, las profesiones aportan conocimiento al poder, y son centrales en el proceso de racionalización al facilitar formas de organización y dominación a través del conocimiento. En Durkheim, que se centra en los aspectos corporativos de las profesiones francesas, las profesiones son condición necesaria para el consensus en la sociedad industrial (función de cohesión social). Las profesiones juegan el doble papel de amortiguar las relaciones entre el Estado y el individuo, a la vez que promueven una ética social superior.

A pesar de que las profesiones tienen en la mayoría de los casos un origen medieval, los primeros intentos de estudiarlas de forma sistemática tienen lugar en este siglo. Hasta después de la segunda guerra mundial los trabajos más importantes son británicos. La obra de Carr-Saunders y Wilson The Professions es el primer intento de estudiar las características centrales de las profesiones. Su discusión teórica sistematizó una visión de las profesiones como organizaciones de expertos aplicando conocimientos esotéricos. Las profesiones habían elaborado un sistema de formación y de control de entrada, y poseían y aplicaban un código de ética. Esta lista de propiedades se convierte en el centro de las definiciones posteriores y caracteriza a la llamada Escuela Definidora. La obra de Carr-Saunders y Wilson combina las dos metodologías características del análisis de profesiones: naturalismo y topologias. El trabajo de Marshall representa la culminación de esta tradición. Para él, las profesiones son signos de un nuevo altruismo que podía transformar el mundo social y político del capitalismo industrial. La Escuela Definidora resalta la importancia de un cuerpo de conocimientos al que el público no puede acceder, la autonomía de las profesiones, y su potencial para contribuir socialmente.

Tras la segunda guerra mundial, y durante más de dos décadas, dominan los académicos americanos y la aproximación funcionalista en el análisis de las profesiones. Destacan los trabajos producidos por Wilensky, 
Greenwood, Barber, Parsons y Goode, entre otros muchos. El elemento común es el énfasis en las características especiales del conocimiento y pericia de los profesionales y su orientación altruística hacia la comunidad. Este grupo de autores configura la llamada Escuela Evolutiva porque entiende a las profesiones como un proceso (proceso de profesionalización). Las profesiones pasan a través de una serie de etapas en su camino hacia la profesionalización. Como profesiones tipo se destacan a la Medicina y al Derecho. Las profesiones son definidas y medidas en términos de características (estructurales y de actitud). Entre las características estructurales destacan la creación de una ocupación a tiempo completo, la creación de asociaciones profesionales, la elaboración de códigos de ética, y la conquista de la autonomía para asegurarse el derecho exclusivo a la práctica profesional y a la evaluación de su trabajo. Las actitudes características son la utilización de la organización profesional como referencia principal, la creencia en el servicio a la comunidad, la creencia en la autorregulación, la idea de llamada, y la creencia en la autonomía como sentimiento de que el profesional debería tomar sus propias decisiones sin presiones externas. En esta aproximación algunas ocupaciones son realmente ocupaciones, mientras que otras son copias aproximadas, todavía en proceso de obtención de algunos de esos atributos.

El concepto de profesionalización representa la unión de las aproximaciones naturalista y tipológica. La profesionalización es vista como un proceso natural (Wilensky, 1964). Pero cuando el concepto de profesionalización parecía ya bien constituido, los cambios políticos de la década de los sesenta transforman el análisis de las profesiones. A partir de ese momento cambia el énfasis en los estudios. Se pasa de la aprobación a la desaprobación, se ataca la idea de profesiones, y se abandona el estudio de las normas profesionales y la relación con los clientes. Ben-David, Hughes, Freidson y Johnson son los primeros autores de la llamada Escuela Revisionista. Los atributos profesionales clásicos ya no son interpretados como componentes de un tipo ideal, sino más bien como instrumentos utilizados por diversas ocupaciones para aumentar su poder con respecto a otros grupos sociales. Se analizan sus actividades en términos políticos, y se destaca la autonomía como el corazón político del profesionalismo. Johnson mantiene que las profesiones no servían las necesidades sociales, sino más bien imponían las definiciones de necesidad y las formas de servirlas en consumidores atomizados. Eliot Freidson mantenía que el dominio y la autonomía son el centro del profesionalismo. Berlant atribuye las estructuras de las profesiones a sus objetivos de monopolio económico.

Los nuevos teóricos al centrarse en el monopolio empujan el centro del debate de las formas de profesionalización a sus funciones. Los nuevos trabajos centrados en el poder califican a las interpretaciones anteriores como ideológicas. El trabajo más representativo en este sentido es el de Magali Larson (The Rise of Professionalism). Para ella, las profesiones están 
explícitamente orientadas hacia el mercado. Son organizaciones que intentan el dominio intelectual y organizativo de áreas de preocupación social. En las sociedades en que las profesiones dependen del mercado, el nacimiento de las profesiones toma forma de proyecto que tiene como objetivo el control del mercado de servicios profesionales. En ese proyecto se utiliza la ideología del profesionalismo para la acción colectiva, buscando el apoyo del Estado para conseguir un refugio en el mercado y la protección ante la competencia. El poder es la pieza clave en los nuevos estudios sobre profesiones. Una gran parte de los trabajos más recientes se centran en la influencia política y cultural de las profesiones (Freidson, Illich, Ehrenreich), las relaciones entre profesiones y élites económicas y el Estado (Johnson, Perkin, Derber), y la relación de la profesión con el mercado y el sistema de clases (Larson, Starr, Brint).

Los estudios sobre profesiones han pasado gradualmente del naturalismo a la teoría. Empezó con estudios de casos y tipologías. Esos estudios llevaron a la idea de profesionalización. El proceso de profesionalización ha sido objeto de varias interpretaciones teóricas: como medio de control de relaciones sociales, como mecanismo de extorsión corporativa; para otros, como canal de progreso individual, y para otros, como forma de ocultar funciones sociales. Todos coinciden en que una profesión era un grupo profesional con una pericia especial, basada en un aprendizaje extenso y en un conocimiento abstracto.

La característica del conocimiento abstracto como fuente de poder es el elemento central de los análisis y teorías más novedosos. Tanto Derber como Freidson y Abbott coinciden en señalar la importancia del conocimiento abstracto como el elemento definidor clave de una profesión. Sólo un sistema de conocimiento gobernado por la abstracción puede redefinir continuamente los problemas y tareas profesionales, defenderlos de los competidores y conquistar nuevos problemas. El conocimiento abstracto es la pieza fundamental para el mantenimiento de la autonomía profesional (Freidson) y las posiciones de poder y privilegio en la sociedad y la organización (Derber). Para Abbott, la abstracción asegura la supervivencia en un sistema de profesiones competitivo.

La innovación más importante del trabajo de Abbott (The System of Professions) es romper con el concepto existente de profesionalización, en el cual el desarrollo de una profesión no era enmarcado en sus relaciones con otras ocupaciones. Para Abbott, el tema central del desarrollo de las profesiones está ligado a las relaciones interprofesionales y al contenido de la actividad profesional. Las características formales de las profesiones no tienen sentido si no se entiende su contexto. $Y$ ese contexto nos hace volver siempre al poder de los sistemas de conocimiento profesional y su capacidad para definir problemas.

Las tendencias más recientes en el análisis y teorización sobre las profesiones se centran en la relación de las profesiones con la estructura 
social, las organizaciones y la política. El tema central es el poder y el privilegio. Los trabajos más novedosos analizan los procesos y mecanismos políticos con los cuales las profesiones conquistan y mantienen posiciones de poder y privilegio en la sociedad, en el mercado y en las organizaciones. Si bien el conocimiento abstracto es un ingrediente necesario, la articulación de proyectos políticos que utilizan ese conocimiento abstracto para la definición de los problemas sociales y sus soluciones (para la definición, en suma, del Estado de Bienestar) es el elemento imprescindible para la ampliación de sus jurisdicciones y el mantenimiento de sus posiciones de poder y privilegio. La relación política con el Estado, su papel en el proceso político, su dinámica y estructura política y el análisis de sus redes de influencia y acción colectiva son centrales en los trabajos teóricos más recientes y significativos sobre las profesiones en la sociedad organizada contemporánea (Abbott, 1989; Brint, 1990, 1992; Derber, 1990; Freddi, 1991; Freidson, 1986; Heinz y Laumann, 1982; Larson, 1977; Laumann y Knoke, 1987; Lauman y Pappi, 1976; Perkins, 1989; Rodríguez, 1991).

\section{LA TEORIA DE LAS ORGANIZACIONES Y LAS PROFESIONES}

La teoría de las organizaciones es un campo de conocimiento interdisciplinar desde sus comienzos. La economía (Smith, Babbage), la ingeniería (Taylor, Gilbreth), la teoría administrativa (Fayol), la psicología (Münsterberg), la ciencia política (Michels), la sociología (Marx, Durkheim, Weber) y la teoría de la decisión (Barnard, Simon) constituyen el sustrato de la moderna teoría de las organizaciones. A lo largo del presente siglo se han sucedido una serie de escuelas sobre teoría de las organizaciones tales como la organización científica, las relaciones humanas, el institucionalismo, la teoría contingente, el análisis estructural, la teoría conductual de la empresa, el modelo de anarquías organizadas y la teoría de la dependencia de recursos (Perrow, 1991; Scott, 1987; Burawoy, 1979; Etzioni, 1964). En la actualidad existen cinco tendencias de vanguardia. La teoría de los costes de transacción (Williamson, 1985) ha recalcado variables económicas a la hora de estudiar los procesos de integración vertical y de adopción de nuevas estructuras organizativas. La teoría ecológica (Hannan y Freeman, 1989; Singh, ed., 1990) estudia poblaciones de organizaciones a lo largo de varias décadas para observar los efectos de la legitimidad y la competencia sobre las tasas de natalidad y mortalidad de organizaciones. La teoría neoinstitucional (Powell y DiMaggio, eds., 1991; DiMaggio, 1988), de carácter eminentemente sociológico, estudia campos o sectores de organizaciones desde el punto de vista de su institucionalización y estructuración (Giddens, 1979, 1982, 1984), con el fin de evaluar los procesos miméticos, coercitivos y normativos que se verifican en el interior del campo de organizaciones. La escuela de las redes organizativas (Laumann y Knoke, 
1989) estudia las relaciones estructurales y conductuales entre los actores organizativos dentro de un campo de organizaciones. Por último, nos encontramos con la nueva escuela comparada de las organizaciones, que resalta la importancia de las diferencias nacionales y regionales en el desarrollo de las distintas formas organizativas y de su difusión internacional (Whitley, 1991; Cole, 1985, 1989; Westney, 1987), o bien las alternativas existentes al modelo de grandes empresas burocratizadas (Piore y Sabel, 1984).

Las profesiones entran a formar parte de algunas de estas escuelas como variables explicativas. Por ejemplo, el estudio de los procesos normativos en el interior de un campo de organizaciones típico de la escuela neoinstitucional se verifica a través del análisis de los grupos profesionales existentes. La escuela comparada de organización también recalca la importancia de estudiar la mentalidad y configuración de las profesiones relevantes para la evolución de las prácticas organizativas. Pero, aparte del papel que los grupos profesionales juegan en los procesos de cambio organizativo, las profesiones se han visto afectadas por el surgimiento de las grandes empresas burocráticas y del Estado. La teoría de las organizaciones ha dedicado gran atención al problema de la asalarización de las profesiones y su impacto sobre la autonomía técnica e ideológica (Guillén, 1990). La asalarización (para algunos autores, proletarización) de las profesiones conduce a una pérdida más o menos amplia del control sobre el proceso y el producto del trabajo profesional, y una expropiación de los valores o del propósito profesional. El trabajo por cuenta ajena en un ambiente burocrático provoca un deterioro progresivo de la autonomía y una sobordinación a los requerimientos de la producción. La especialización del conocimiento profesional y la irrupción de nuevas tecnologías, sin duda, contribuyen a esa pérdida de autonomía (Derber, ed., 1983).

Pero la moderna teoría de las organizaciones nos presenta una versión distinta de los hechos. Para algunos autores (Mintzberg, 1987; Perrow, 1991; Freidson, 1986; Scott, 1987), las profesiones encuentran un medio favorable en las organizaciones complejas tales como las empresas y el Estado. Estas organizaciones contratan a profesionales como economistas, médicos, abogados, ingenieros, maestros, etc., para realizar ciertos tipos de tareas caracterizadas por un elevado número de casos excepcionales y por un modo relativamente poco estructurado de buscar una solución para esas excepciones. La discreción profesional es, por tanto, elevada en estas ocupaciones. Las organizaciones que poseen muchos de esos puestos reservados a profesionales (por ejemplo, hospitales, ministerios, firmas de consultoría) suelen desarrollar estructuras orgánicas, flexibles y policéntricas para conseguir la coordinación de los distintos individuos (profesionales) y unidades organizativas. Perrow (1991) sostiene que el problema no consiste en determinar si el profesional trabaja por cuenta ajena o no, sino más bien de cuánto poder discrecional disfruta. Las organizaciones disponen 
de dos vías para evitar que sus empleados gocen de demasiada discreción a la hora de realizar su trabajo: desarrollar lealtad o profesionalizar. Ambas hacen a los empleados más predecibles en su comportamiento. Los profesionales se ajustan bien a las burocracias porque, dada su formación vocacional, tienen reglas y normas de comportamiento adquiridas. De ese modo puede anticiparse y controlarse el resultado de una tarea que es en principio discrecional. Mintzberg (1987) postula que las burocracias profesionales consiguen la coordinación a través de la estandarización de las destrezas técnicas de los profesionales, que, a su vez, se alcanza durante el período de formación universitaria.

La teoría de la proletarización de las profesiones olvida que existen diferencias entre las distintas organizaciones para las que trabajan los profesionales. Hall (1968) propone la distinción entre organizaciones autónomas, heterónomas y departamentos profesionales. Una organización es autónoma si está compuesta únicamente por profesionales, que constituyen la jerarquía administrativa de la organización. Los despachos o consultorías de abogados o economistas y las clínicas médicas privadas de pequeño tamaño son ejemplos típicos. Las organizaciones beterónomas difieren del tipo anterior en que los profesionales que trabajan en ellas están sujetos a una jerarquía externa no-profesional. Una escuela pública, las sucursales de un banco o el gran hospital burocratizado son algunos ejemplos de este tipo. Los departamentos profesionales dentro de organizaciones más grandes son un tipo especial para el que no resulta fácil determinar a priori si los profesionales ejercen control sobre su trabajo o no. El debate en torno al conflicto entre jerarquías administrativas y profesionales (entre auoridad formal y autoridad del conocimiento experto) tiene una historia prolongada. Parsons (1947) fue quien lo popularizó, si bien el problema prosigue en nuestros días. Acontecimientos tales como la rebelión de los médicos en los hospitales confirman la actualidad de este tipo de conflicto organizativo.

Las nuevas tendencias en sociología de las profesiones emplean a fondo ciertos aspectos de la teoría de las organizaciones. Abbott (1988) emplea en su aproximación sistémico-ecológica elementos organizativos, y su noción de que el cambio profesional se verifica a través de luchas interprofesionales requiere el estudio de las organizaciones asociativas profesionales. Freidson (1986) recalca el papel del conocimiento experto y de las rutinas profesionales, precisamente los aspectos que más preocupan a los sociólogos de las organizaciones a la hora de estudiar el comportamiento de los profesionales en las organizaciones complejas. Se viene produciendo, por tanto, un acercamiento entre la sociología de las profesiones y la teoría de las organizaciones. Este campo se nos presenta como uno de los más prometedores en sociología. 


\section{BIBLIOGRAFIA}

Аввотт, Andrew (1988): The System of Professions: An Essay on the Division of Expert Labor, Chicago: The University of Chicago Press.

Ben-David, Joseph (1963): «Professions in the Class System of Present Day Societies», Current Sociology, 12: 247-298.

Berlant, Jeffrey (1975): Professions and Monopoly, Berkeley: Universiy of California Press.

BlEDSTEIN, Burton (1976): The Culture of Professionalism, Nueva York: Norton.

BRINT, Steven (1990): «Rethinking the policy influence of experts: From general characterizations to analysis of variation», Sociological Forum, 5 (3): $361-385$.

- (1992): Retainers, Merchants and Priests: A Political Economy of the Professional America, Berkeley: University of California Press.

BurAwOY, Michael (1979): «The Anthropology of Industrial Work», Annual Review of Antbropology, 8: 231-266.

Burrage, M., y Torstendahl, R. (eds.) (1990): Professions in Theory and History, Londres: Sage.

COLE, Robert E. (1985): «The macropolitics of organizational change: A comparative analysis of the spread of small-group activities», Administrative Science Quarterly, 30 (4) (diciembre): 560-585.

- (1989): Strategies for Learning: Small-Group Activities in American, Japanese, and Swedish Industry, Berkeley, California: University of California Press.

Coleman, James (1974): Power and the Structure of Society, Nueva York: Norton.

- (1986): Individual Interests and Collective Action, Cambridge: Cambridge University Press.

De Miguel, Jesús (1990): El mito de la sociedad organizada, Barcelona: Península.

DERBER, Charles (ed.) (1983): The Project on Professionals: Report to Responding Organizations, Chesnut Hill, Massachusetts: Boston College.

- (1982): Professionals as Workers: Mental Labor in Advanced Capitalism, Boston: G. K. Hall.

Derber, A.; SChwartz, W., y Magras, Y. (1990): Power in the Highest Degree, Nueva York: Oxford University Press.

DiMAgGio, Paul J. (1986): «Structural Analysis of Organizational Fields: A Blockmodel Approach», Research in Organizational Behavior, 8: 335-370.

- (1988): «Interest and Agency in Institutional Theory», en Lynne G. Zucker (ed.), Institutional Patterns and Organizations: Culture and Environment, Cambridge, Massachusetts: Ballinger, pp. 3-21.

Etzioni, Amitai (1964): Moderns Organizations, Englewood Cliffs, New Jersey: PrenticeHall.

Fredd, Giorgio, y Warner BJörkman, J. (1989): Controlling Medical Professionals, Londres: Sage.

FreIDSON, Eliot (1986): Professional Powers: A Study of the Institutionalization of Formal Knowledge, Chicago: The University of Chicago Press.

Giddens, Anthony (1979): Central Problems in Social Theory, Londres: Macmillan.

- (1982): Profiles and Critiques in Social Theory, Berkeley, California: University of California Press.

- (1984): The Constitution of Society, Berkeley, California: University of California Press.

GOODE, W. J. (1960); «Encroachment, charlatanism, and the emerging profession», American Sociological Review, 25: 902-914.

GOUlDNER, Alvin (1981): The Future of Intellectuals and the Rise of the New Class, Nueva York: Oxford University Press.

GuILLÉN, Mauro F. (1990): «Profesionales y burocracia: Desprofesionalización, proletarización y poder profesional en las organizaciones complejas», REVISTA ESPAÑOLA DE INVESTIGACIONES SOCIOLOGICAS, 51 (julio-septiembre): 35-51.

Hall, Richard H. (1968): «Professionalization and bureaucratization», American Sociological Review, 33 (1): 92-104. 
Hannan, Michael T., y Freeman, John (1989): Organizational Ecology, Cambridge, Massachusetts: Harvard University Press.

Haugh, M. R. (1976): «The Erosion of Professional Authoriry», Healtb and Society, The Milbank Memorial Fund Quarterly, 54: 8-104.

Heinz, J. P., y Laumann, E. O. (1982): Chicago Lawyers, Nueva York: Rusell Sage.

Johnson, Terry (1967): Professional Powers, Londres: Macmillan.

KNOKE, David (1990): Political Networks: The Structural Perspective, Nueva York: Cambridge University Press.

LARSON, Magali S. (1977): The Rise of Professionalism, Berkeley, California: University of California Press.

Laumann, Edward O., y KNoKe, David (1989): The Organizational State: Social Cboice in National Policy Domains, Madison, Wisconsin: The University of Wisconsin Press.

Laumann, Edward O., y PAPPI, F. (1976): Networks of Collective Action, Nueva York: Academic Press.

MintzBeRG, Henry (1987): La estructuración de las organizaciones, Barcelona: Ariel.

Parsons, Talcott (1947): «Introduction», en Max WeBER, Theory of Social and Economic Organization, Nueva York: Oxford University Press, pp. 58-60.

- (1954): «The Professions and Social Structure», en Essays in Sociological Tbeory, Nueva York: Free Press, pp. 34-95.

Perrow, Charles (1990): Sociología de las Organizaciones, 3." ed., Madrid: McGraw-Hill/ Interamericana.

PIORE, Michael, y SABel, Charles F. (1984): The Second Industrial Divide: Possibilities for Prosperity, New York: Basic.

Powell, Walter W., y DiMagGio, Paul J. (eds.) (1991): The New Institutionalism in Organizational Analysis, Chicago: The University of Chicago Press.

Rodriguez, Josep A. (1991): The Politics of Medicine in Spain, New Haven: Yale University.

- (1992): «Struggle and Revolt in the Spanish Health Policy Process: The Changing Role of the Medical Profession», International Journal of Healtb Services, 22: 19-44.

Scort, W. Richard [1981] (1987): Organizations: Rational, Natural, and Open Systems, 2.a ed., Englewood Cliffs, New Jersey: Prentice-Hall.

Singh, Jitendra (ed.) (1990): Organizational Evolution: New Directions, Newbury Park, California: Sage.

STARR, Paul (1982): The Social Transformation of Medicine, Nueva York: Basic.

Tilly, Charles (1978): From Mobilization to Revolution, Reading, Massachusetts: AddisonWesley.

- (1992): Coerción, Capital y Estados Europeos, 990-1990, Madrid: Alianza Universidad.

Torstendahl, R., y BurRage, M. (eds.) (1990): The Formation of Professions: Knowledge, State and Strategy, Beverly Hills, California: Sage.

Weber, Max [1904] (1958): The Protestant Ethic and the Spirit of Capitalism, New York: Charles Scribner's Sons.

- (1978): Economy and Society: An Outline of Interpretive Sociology, 2 vols, editado por Guenther Roth y Claus Wittich, Berkeley, California: University of California Press.

WestNey, D. Eleanor (1987): Imitation and Innovation: The Transfer of Western Organizational Patterns to Meiji Japan, Cambridge, Massachusetts: Harvard University Press.

WhITLEY, Richard (1991): «Societies, Firms and Markets: The Social Restructuring of Market Economies», trabajo presentado en la «Conference on Organizational Theory at the Crossroads: European Contributions to the Science of Organizations», INSEAD, Fontainebleau, Francia, 29-30 de noviembre de 1991.

WILENSKY, Harold L. (1964): «The professionalization of everyone?», American Journal of Sociology, 70 (2) (septiembre): 137-158.

Williamson, Oliver E. (1985): The Economic Institutions of Capitalism: Firms, Markets, Relational Contracting, New York: Free Press. 